\title{
Highly Sensitive Bend Sensor Based on Bragg Grating in Eccentric Core Polymer Fiber
}

\author{
Xianfeng Chen, Chi Zhang, David J. Webb, Kyriacos Kalli, and Gang-Ding Peng
}

\begin{abstract}
We report on an optical bend sensor based on a Bragg grating inscribed in an eccentric core polymer optical fiber. The device exhibits the strong fiber orientation dependence, the wide bend curvature range of $\pm 22.7 \mathrm{~m}^{-1}$ and high bend sensitivity of $63 \mathrm{pm} / \mathrm{m}^{-1}$.
\end{abstract}

Index Terms-Bragg grating, polymer optical fiber, bend sensor

\section{INTRODUCTION}

$\mathrm{O}$ VER the last decade, fiber Bragg gratings (FBGs) and long-period gratings (LPGs) have been widely developed as devices for optical sensing and communications [1], [2]. To date, a number of bend sensors have been realized using LPG structures to measure curvature [3]-[5]. Despite high bend sensitivity, LPGs exhibit several disadvantages, including broad resonance which limits measurement accuracy, measurement in transmission only and relatively long device size. In contrast, with FBG-based bend sensors the small size and measurement-in-reflection make them ideal as point-probe sensors [6]-[9]. To date, all the work on fibre grating based bend sensor have concerned silica optical fiber.

In the past few years, FBGs in the polymer optical fibers (POFs) have begun to attract some interest as they offer the prospect of some significant advantages over the silica-based devices for certain applications [10]. Compared with the silica fiber, the Young's modulus of the polymer fiber is much smaller and the failure strain of the polymer fiber is much larger [11]-[14]. In addition, the design flexibility of the POF facilitates the creation of asymmetric structure permitting the curvature measurement with the enhanced bend sensitivity [15].

This letter reports on a bend sensor based on a Bragg grating in an eccentric core polymer optical fiber (ecPOF). This sensor displays the characteristics such as the high bend sensitivity, the

Manuscript received October 29, 2009. This work was supported by EU FP7 Project PHOSFOS (PHOtonic Skins For Optical Sensing) and the UK Engineering and Physical Sciences Research Council.

X. Chen, C. Zhang, D. J. Webb are with School of Engineering and Applied Science, Aston University, Birmingham B4 7ET, United Kingdom (e-mail: x.f.chen@aston.ac.uk).

K. Kalli is with Nanophotonics Research Laboratory, Cyprus University of Technology, Cyprus.

G. D. Peng is with School of Electrical Engineering, University of South Wales, Australia. wide curvature measurement range and the strong directional dependence.

\section{GRATING FABRICATION AND SPECTRUM MEASUREMENT}

The POF used in our experiments is a single mode step index polymethylmethacrylate (PMMA) based fibre with the cross section image displayed in Fig. 1. Two cores are offset from the fiber neutral axis. The diameters of the core and the cladding are $8.1 \mu \mathrm{m}$ and $230 \mu \mathrm{m}$, respectively.

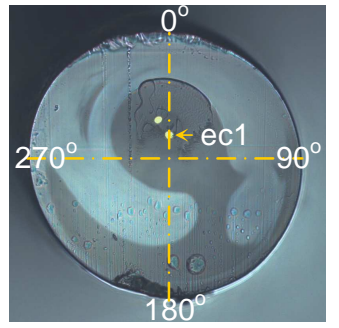

Fig. 1. Microscope image of POF cross section marked with the orientations.

In our work only the core marked as "ec1" with an offset of $24 \mu \mathrm{m}$ from the neutral axis was selected to write FBG. Small masking tapes were attached to the fiber to indicate its orientations. As shown in Fig. 1 , the $0^{\circ}-180^{\circ}$ orientation was defined where the ec1-core and the fiber axis lie along the same line.

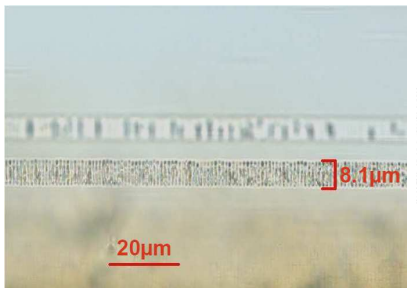

(a)

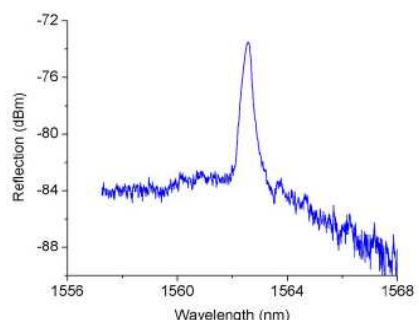

(b)
Fig. 2. (a) FBG fringes observed under microscope, (b) the measured Bragg grating spectrum when the light coupled into ec1-core.

The polymer FBG was written using a CW helium-cadmium laser with an output wavelength of $325 \mathrm{~nm}$, a beam diameter of $1.8 \mathrm{~mm}$ and a power of $30 \mathrm{~mW}$. With the polymer fiber mounted horizontally in a v-groove, the laser beam was focused vertically down onto the fiber core using a cylindrical lens with $10 \mathrm{~cm}$ focal length and was scanned $4 \mathrm{~mm}$ 
along a phase mask with $1057 \mathrm{~nm}$ period to generate a periodic index modulation in the ec1-core, which was confirmed by the microscope and displayed in Fig. 2a.

The FBG spectrum was measured by the butt-coupling technique in conjunction with a broadband light source (Thorlabs, Broadband ASE light source with output power of $-25 \mathrm{dBm}$ over $1.53-1.61 \mu \mathrm{m}$ wavelength range), a $3-\mathrm{dB}$ coupler and an optical spectrum analyzer (OSA, HP86142A). The light was launched to the cleaved polymer fibre end by adjusting the three-dimensional stage. In order to eliminate the back reflection from the fibre ends and to minimize any Fresnel reflections, the refractive index matching gel was applied to the fibre ends. The coupling efficiency of $5 \mathrm{~dB}$ was achieved. The grating reflection as monitored by the OSA with $0.06 \mathrm{~nm}$ resolution bandwidth is plotted in Fig. 2b, displaying a Bragg wavelength of $1562.5 \mathrm{~nm}$, a FWHM of $0.49 \mathrm{~nm}$ and a reflective power above the noise level of $10.5 \mathrm{~dB}$.

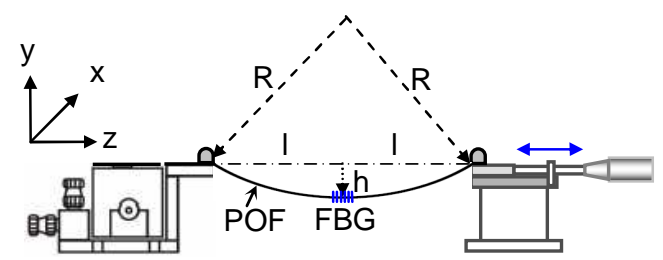

Fig. 3. Schematic of the experimental setup for characterizing the FBG based sensor. $\mathrm{R}$ is the bend radius and $\mathrm{h}$ is the displacement.

\section{EXPERIMENTS AND RESULTS}

The strain of a silica FBG is well known [16], however it is not the case of this kind of polymer FBG. The strain response will therefore be described here firstly. As shown in Fig.3, the polymer fiber was hung between two translation stages with two ends clamped (section length is $90 \mathrm{~mm}$ ) with the Bragg grating at its center.

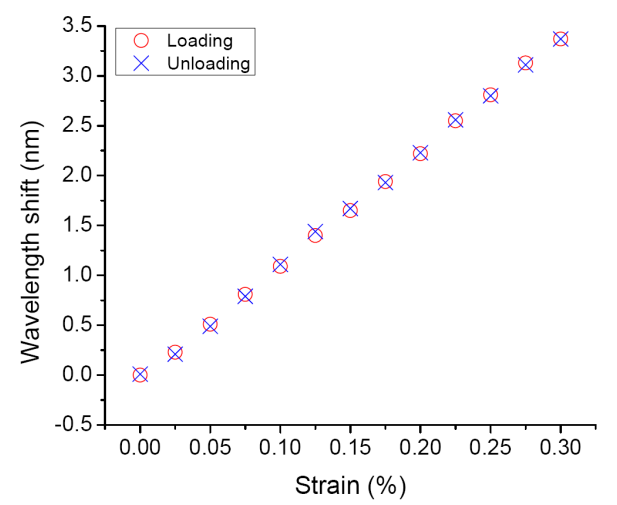

Fig. 4. Strain response of Bragg grating in ecPOF.

The ecPOF was stretched by driving the micrometer screw of right-hand stage along ' $+z$ ' direction up to $0.3 \%$ after which the translation stage was returned to its starting position incrementally. We can see from the Fig. 4 that the strain loading data exhibit good linearity with no hysteresis apparent when the ecPOF is unloaded, which is the same as the behavior reported for POF [11]. The strain sensitivity of Bragg grating in this ecPOF is $1.12 \mathrm{pm} / \mu \varepsilon$. It is similar as the value for the silica FBG which is $1.15 \mathrm{pm} / \mu \varepsilon$ at $1550 \mathrm{~nm}$ wavelength [16].

To minimize the temperature cross-sensitivity, all the sensing experiments were carried out at a constant room temperature of $22^{\circ} \mathrm{C} \pm 0.3^{\circ} \mathrm{C}$. Control of the bend was achieved by the translation of the right-hand end of the polymer fiber, which was driven by a micrometer screw along '- $z$ ' direction resulting in a bend that lay in the vertical plane, i.e., $y$-z plane. As the inherent flexibility of POFs, the precaution was taken to avoid fibre twist. Two transparent plastic plates were setup standing along the vertical plane with a parallel gap to keep the polymer fibre bending within this gap. The bending curvature $\mathrm{C}$ is given by,

$$
C=\frac{1}{R}=\frac{2 h}{h^{2}+l^{2}}
$$

where $\mathrm{h}$ is the displacement in the vertical plane from the straight position and 1 is the half length of bend section.

Experiments were conducted to determine the grating spectral responses to the curvature variations at different fiber orientations. Small masking tapes attached to the polymer fiber were used to indicate the fiber rotations with respect to the vertical plane. The bend experiments were curried out for the four orientations individually. For each rotational orientation, the Bragg peak shift was monitored for a curvature range from 0 to $22.7 \mathrm{~m}^{-1}$. Strong directional bend sensitivity characteristics were observed when bending was applied at different orientations. With the ecPOF fixed at $0^{\circ}$ orientation, the Bragg peak moved to the short wavelength side (Fig. 5a) whereas the peak showed a red-shift for the $180^{\circ}$ orientation case (Fig. 5b).

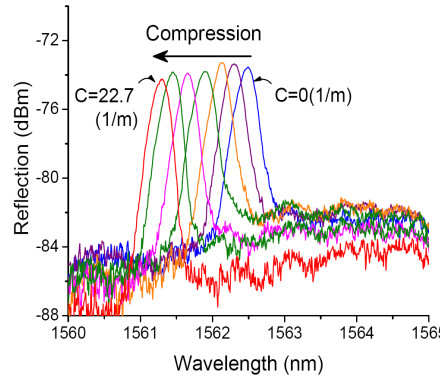

(a)

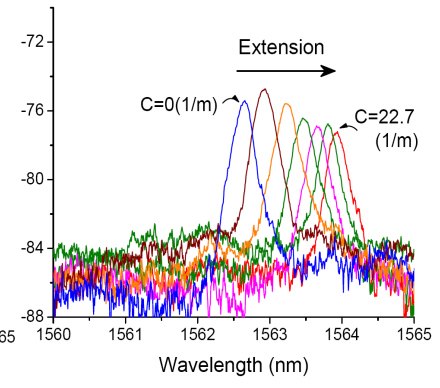

(b)
Fig. 5. Spectral response of polymeric FBG experiencing bending at fiber orientations: (a) $0^{\circ}$ (Compression), (b) $180^{\circ}$ (Extension).

The Bragg wavelength shifts against curvature for four orientations are quantified in Fig. 6. It is clear that the bend responses are directionally sensitive and all the responses exhibit near linear features. It has been demonstrated that the maximum bend response occurred at $0^{\circ}$ and $180^{\circ}$ orientation where the eccentric FBG and fiber neutral axis lie in the bend plane. In addition, the $0^{\circ}$ and $180^{\circ}$ orientations showed the opposite bend response, the sign of the wavelength shift depending on whether bending resulted in a compression $\left(0^{\circ}\right)$ or 
extension $\left(180^{\circ}\right)$ along the core. For $90^{\circ}$ and $270^{\circ}$ orientations, the grating and fiber axis lay in the same approximately neutral bending plane, therefore the minimum response was obtained, showing, for low curvatures, a bend insensitive property.

The core is offset from the neutral axis, inducing the required asymmetry in the device and therefore inducing the bend sensitivity: with a $22.7 \mathrm{~m}^{-1}$ curvature compression bend $\left(0^{\circ}\right.$ orientation), a blue wavelength shift of $-1.26 \mathrm{~nm}$ was obtained; with the same magnitude of extension bend $\left(180^{\circ}\right.$ orientation $)$, there was a red wavelength shift of $+1.42 \mathrm{~nm}$; with the fiber orientation rotated to $90^{\circ}$ or $270^{\circ}$, the wavelength shift was no more than $\pm 0.18 \mathrm{~nm}$. The departure from linearity observed in Fig. 6 and the slight sensitivity to bending observed at the $90^{\circ}$ and $270^{\circ}$ positions for larger curvatures may be caused by non-perfect circular geometry of POF cladding, misalignment of the grating axis with the $0^{\circ}$ bend plane or a slight twist of the POF during bend, in addition, the angle deviation of $\pm 2.5^{\circ}$ when the masking tapes attached to the fiber. The best-fit straight lines to the experimental data in Fig. 6 at $180^{\circ}$ and $0^{\circ}$ give sensitivities of $+63 \mathrm{pm} / \mathrm{m}^{-1}$ and $-56 \mathrm{pm} / \mathrm{m}^{-1}$, respectively. The bend sensitivity here is as high as that of the sensor based on multiple-core silica fiber reported by Askins et al. [9]. In this work, the grating axial-offset is $24 \mu \mathrm{m}$ which is 8 times that used in the bend sensor based on an offset-FBG [6], but the maximum bend sensitivity here of $63 \mathrm{pm} / \mathrm{m}^{-1}$ is 80 times higher than that of the device.

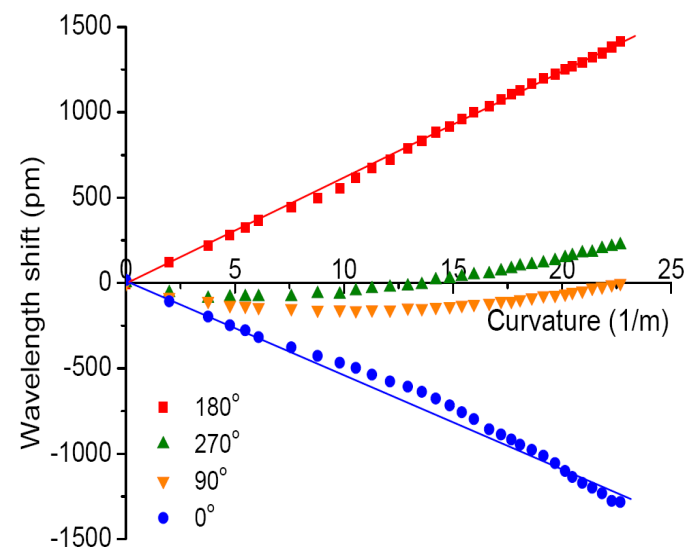

Fig. 6. Wavelength shifts of the eccentric polymeric FBG against curvatures at four different orientations. The symbols show the experimental data and lines depict the best-fit lines.

\section{CONCLUSION}

We have demonstrated a curvature sensor based on a Bragg grating inscribed in an ecPOF. This polymeric grating sensor is capable of measuring the magnitude and orientation of the applied bend. The maximum bend sensitivity achieved as high as $63 \mathrm{pm} / \mathrm{m}^{-1}$. It is clear that the bend sensitivity depends on the distance of the offset core from the neutral axis, the larger the core displacement the higher the bend sensitivity. One issue with devices of this kind is how to produce a simple means of coupling light from a conventional fiber into the eccentric core. The POF production process [15] in principle allows the development of fiber in which the core alternates from being concentric to being eccentric along the fiber length. This offers the possibility of coupling to the POF where the core is concentric but still having an eccentric sensing region. Alternatively, permanent connections between silica fiber and polymer fiber can be realized by gluing the fibers, which permits coupling to the core in any position.

\section{ACKNOWLEDGMENT}

The authors acknowledge the financial support of EU FP7 Project PHOSFOS (PHOtonic Skins For Optical Sensing) and the UK Engineering and Physical Sciences Research Council.

\section{REFERENCES}

[1] G T. Erdogan, "Fiber grating spectra," J. Lightwave Technol., 15, 1277-1294, 1997.

[2] A. Othonos and K. Kalli, Fiber Bragg Gratings: Fundamentals and Applications in Telecommunications and Sensing, Artech House Inc., 1999.

[3] H. J. Patrick, "Self-aligning, bipolar bend transducer based on long period grating written in eccentric core fiber," Electron. Lett., 36(21), 1763-1764, 2000.

[4] C. C. Ye, S. W. James, and R. P. Tatam, "Simultaneous temperature and bend sensing with long-period fiber gratings," Opt. Lett., 25(14), 1007-1009, 2000.

[5] D. Zhao, K. Zhou, X. Chen, L. Zhang, I. Bennion, G. Flockhart, W. Macpherson, J. Barton, and J. Jones, "Implementation of vectorial bend sensors using long-period gratings UV-inscribed in special shape fibers," Meas. Sci. Technol., 15, 1647-1650, 2004

[6] A. Martinez, Y. Lai, M. Dubov, I. Y. Khrushchev, and I. Bennion, "Vector bending sensors based on fiber Bragg gratings inscribed by infrared femtosecond laser," Electron. Lett., 41(8), 472-474, 2005.

[7] Z. Yong, C. Zhan, J. Lee, and S. Z. Yin, "Multiple parameter vector bending and high temperature sensors based on asymmetric multimode fiber Bragg gratings inscribed by an infrared femtosecond laser," Opt. Lett., 31 (12), 1794-1796, 2006.

[8] G. A. Cranch, G. Flockhart, W. MacPherson, J. Barton, and C. Kirkendall, "Ultra-high-sensitivity two-dimensional bend sensor," Electron. Lett., 42(9),1516-1517, 2006.

[9] C. G. Askins, G. A. Miller, and E. J. Friebele, "Bend and twist sensing in a multiple-core optical fiber," in Optical Fiber Communication Conference, paper OMT3, 2008.

[10] Z. Xiong, G. D. Peng, B. Wu, and P. L. Chu, "Highly tunable Bragg gratings in single-mode polymer optical fibers," IEEE Photon. Technol. Lett., 11(3), 352-354, 1999.

[11] H. B. Liu, H. Y. Liu, G. D. Peng, and P. L. Chu, "Strain and temperature sensor using a combination of polymer and silica fiber Bragg gratings," Opt. Commun., 219, 139-142, 2003.

[12] H. Dobb, D. J. Webb, K. Kalli, A. Argyros, M. C. J. Large, and M. A. Eijkelenborg, "Continuous wave ultraviolet light-induced fiber Bragg gratings in few- and single-mode microstructured polymer optical fibers," Opt. Lett., 30(24), 3296-3298, 2005.

[13] K. Carroll, C. Zhang, D. J. Webb, K. Kalli, A. Argyros, and M. Large, "Thermal response of Bragg gratings in PMMA microstructured optical fibers," Opt. Express, 15 (14), 8844-8850, 2007.

[14] X. Chen, C. Zhang, D. J. Webb, R. Suo, G.-D. Peng, and K. Kalli, "Optical bend sensor for vector curvature measurement based on Bragg grating in eccentric core polymer optical fibre", Proc. SPIE Vol. 7503 750327, 2009.

[15] M. A. Van Eijkelenborg, A. Argyros, G. Barton and I. M. Bassett, "Recent progress in microstructured polymer optical fiber fabrication and characterisation", Opt. Fiber Technol., 9, 199-209, 2003.

[16] Y.-J. Rao, "In-fiber Bragg gratings,". Meas. Sci. Technol. 8 355-375, 1997. 